\title{
A Prática de um Programa Preventivo Melhora a Capacidade Funcional de Membros Inferiores em Atletas Jovens de Handebol Feminino
}

\author{
Nadine Castro Portuguez, ${ }^{1}$ Simone Lara, ${ }^{2}$ Lilian Pinto Teixeira, ${ }^{1}$ Vinícius Jardim Oliano ${ }^{1}$
}

\begin{abstract}
RESUMO
O objetivo deste estudo foi analisar o efeito cumulativo de oito semanas de um programa preventivo associado ao treinamento convencional de handebol sobre a capacidade funcional de membros inferiores em atletas jovens de handebol feminino. Este estudo experimental incluiu uma amostra por conveniência de 17 atletas com idade de 12 a 16 anos, integrantes de um time de handebol feminino amador. As jovens foram divididas em dois grupos: o grupo intervenção (GI), integrando as atletas que praticaram o programa preventivo associado ao treino regular do handebol $(n=9)$, e o grupo controle (GC), cujas jovens praticaram apenas o treinamento esportivo na equipe $(n=8)$. Foi aplicado um questionário inicial, mensurando os dados antropométricos, e a capacidade funcional dos membros inferiores foi avaliada por meio do Single Leg Hop Test, Timed Hop Test e Side Hop Test. O programa consistiu de aproximadamente 20 minutos de exercícios de aquecimento, força, agilidade e pliometria e equilíbrio, antes do treino regular do esporte, duas vezes por semana, durante oito semanas, totalizando 16 sessões. O Gl apresentou melhorias significativas pós-intervenção em todos os testes realizados ( $<<0,05$ ), em ambos os membros inferiores, diferentes do GC, no qual os resultados foram mais limitados. Oito semanas de treinamento de um programa preventivo melhoraram significativamente a capacidade funcional de jovens atletas de handebol feminino, indicando que este tipo de intervenção é capaz de modificar positivamente os fatores de risco para o desenvolvimento de lesões de membros inferiores destas atletas.
\end{abstract}

Palavras-chave: Atletas. Esportes. Fatores de risco. Lesões esportivas.

THE PRACTICE OF AN INJURY PREVENTION PROGRAM IMPROVES FUNCTIONAL CAPACITY OF LOWER LIMBS IN YOUNG FEMALE HANDBALL PLAYERS

\begin{abstract}
The aim of this study was to analyze the cumulative effects of an eight-week injury prevention program in addition to conventional handball training on functional capacity of lower limbs in young female handball players. This experimental study includes a convenience sample of 17 athletes aged 12 to 16 years, members of an amateur handball team. Athletes were randomly divided into two groups, the intervention group (IG), composed by the athletes whose practiced the prevention program associated to the regular handball training $(n=9)$, and the control group (CG) whose young only practiced the team sport training $(n=8)$. It was applied an initial questionnaire, measured anthropometric data and the functional capacity was evaluated though Single Leg Hop Test, Timed Hop Test and Side Hop Test. Training program was about 20 minutes of warm up, strength, agility, plyometric and balance exercises, before the regular sport training, twice a week, along eight weeks, totalizing 16 sessions. IG showed significative improvement pos-intervention in all tests $(p<0,05)$, of both lower limbs, unlike CG which results were limited. Eight weeks of an injury prevention program training improved significantly the functional capacity of young female handball players, showing that this kind of intervention is capable to modify positively these athletes' lower limbs injury risk factors.
\end{abstract}

Keywords: Athletes. Athletic injuries. Risk factors. Sports.

\footnotetext{
Universidade Federal do Pampa (Unipampa), Campus Uruguaiana/RS, Brasil.

Autora correspondente. Universidade Federal do Pampa (Unipampa), Campus Uruguaiana/RS. BR 472, Km 592, $97508-000$, Brasil, Cx. Postal 118. http://lattes.cnpq.br/5130937270549081. https://orcid.org/0000-0003-0745-4964. simonelara@unipampa.edu.br
} 


\section{INTRODUÇÃO}

O handebol é um esporte olímpico de equipe, que possui ritmo acelerado com características dinâmicas, as quais abrangem transições de ataque-defesa com o objetivo de marcar gols. Ao longo da partida as atletas são submetidas a repetidos esforços de alta intensidade com duração variável (WAGNER et al., 2014; SPIESZNY; ZUBIK, 2018).

Diversos fatores influenciam o desempenho em quadra, haja vista que o handebol é uma modalidade complexa e multifatorial. Desse modo, as atletas necessitam de uma boa coordenação dos movimentos para correr, pular, mudar de direção, bloquear, entre outros gestos específicos do esporte (WAGNER et al., 2014). As técnicas realizadas (passes, arremessos, saltos), bem como comportamentos técnicos e táticos adequados (por exemplo, manuseio da bola, ações defensivas individuais, etc.) exigem que a atleta desenvolva altos valores de potência em um tempo muito curto (SPIESZNY; ZUBIK, 2018).

Tendo em vista as exigências do esporte, é necessário que a atleta potencialize a sua performance funcional em quadra, não somente para melhorar o desempenho no que respeita às habilidades relacionadas ao salto, corrida e resistência aeróbica (MAZUREK et al., 2018), mas também para reduzir a probabilidade do desenvolvimento de lesões. Cabe destacar que as taxas de lesões nessa modalidade são expressivas, especialmente envolvendo atletas do sexo feminino, como apresentado no estudo proposto por Giroto et al. (2017). O autor analisou 339 atletas de elite de handebol, de ambos os sexos, durante dois campeonatos brasileiros, e identificou 312 novas lesões em 201 atletas, com o tornozelo e o joelho sendo as articulações mais afetadas. As atletas do sexo feminino apresentaram maiores taxas de lesões quando comparadas com o sexo masculino $(66,7 \%$ e $33,3 \%$, respectivamente).

Com base nas taxas significativas de lesão apresentadas, estratégias de prevenção de lesão associadas ao treinamento regular de equipes de handebol são de suma importância. Nesse sentido, Achenbach et al. (2018) evidenciaram que um programa de prevenção de lesão foi capaz de reduzir lesões graves em membros inferiores, em uma amostra formada por atletas jovens de handebol, e deve, portanto, ser incluído na rotina prática e na educação dos treinadores de equipes. Ainda, na perspectiva de prevenção, o treinamento pliométrico e de força muscular mos- tram-se importantes para aprimorar o desempenho de saltos e corrida do atleta em quadra (SPIESZNY; ZUBIK, 2018).

Embora dados da literatura apontem para a efetividade de programas de prevenção em diversos esportes - como o futebol (SILVERS-GRANELLI et al., 2017) e o handebol (ACHENBACH et al., 2018) - grande parte dos estudos evidenciam resultados dessas intervenções em times adultos (ELERIAN; EL-SAYYAD; DORGHAM , 2019), e poucos trazem dados para atletas jovens, e, especialmente, do sexo feminino (THOMPSON et al., 2017).

Nesse contexto, há de se considerar que, no atleta jovem, que ainda está em processo de crescimento/desenvolvimento (particularmente rápido no período do salto pubertário), as cartilagens de crescimento presentes nas extremidades dos ossos longos são vulneráveis a lesões por sobrecarga mecânica (OLIVEIRA, 2009). Ademais, essa imaturidade musculoesquelética, associada com um rápido aumento na carga de treinamento, podem ser fatores que predispõem o desenvolvimento de lesões por uso excessivo em atletas jovens (DIFIORI et al., 2014). De fato, atletas jovens do sexo feminino apresentam maior risco de desenvolvimento de lesão musculoesquelética em membros inferiores, quando comparados aos atletas do sexo masculino da mesma faixa etária, devido a diferenças biomecânicas e de controle motor entre os sexos (STEFFEN et al., 2016).

Considerando esses fatores, é de fundamental importância o planejamento de estratégias de prevenção de lesão direcionados a atletas jovens. $O$ objetivo desse estudo, portanto, foi analisar o efeito cumulativo de oito semanas de um programa preventivo associado ao treinamento convencional de handebol sobre a capacidade funcional de membros inferiores em atletas jovens de handebol feminino.

\section{MATERAIS E MÉTODOS}

\section{Amostra}

Trata-se de um estudo experimental, descritivo e quantitativo, o qual incluiu uma amostra por conveniência composta por atletas com idade de 12 a 16 anos, integrantes de um time de handebol feminino amador, em um município do interior do Rio Grande do Sul, Brasil, que estivessem em treinamento regular de handebol por, no mínimo, três meses. Optou-se por essa forma de seleção amostral pelo fato de que a equipe incluída no estudo faz parte da escola pública que mais participa de competições do município. Foram adotados os seguintes critérios de exclusão: 
atletas em afastamento do time por qualquer lesão musculoesquelética, atestada por meio de laudo médico e/ou pós-operatório imediato, atletas com participação inferior a $75 \%$ no programa preventivo, atletas que não participaram da reavaliação do estudo e atletas que desistiram de participar da equipe.

Todos os critérios éticos foram estabelecidos, em conformidade com a resolução 466 do Conselho Nacional de Saúde, observando-se que os responsáveis legais pelas atletas assinaram o termo de consentimento livre e esclarecido (TCLE) e as atletas assinaram o termo de assentimento. $O$ estudo foi aprovado pelo Comitê de Ética em Pesquisa institucional (registro 1.473.781, CAAE 53365716.5.0000.5323).

Inicialmente 29 atletas foram incluídas, sendo alocadas, por meio de um sorteio, em dois grupos: o grupo intervenção (GI), integrando as atletas que praticaram o programa preventivo associado ao treino regular do handebol $(n=14)$, e o grupo controle (GC), cujas jovens praticaram apenas o treinamento esportivo no time $(n=15)$. Doze delas, contudo, foram excluídas, das quais 6 desistiram de participar da equipe e as outras 6 tiveram participação inferior a 75\% no programa preventivo, totalizando ao final 17 jogado$\operatorname{ras}(\mathrm{Gl}=9$ atletas, $\mathrm{GC}=8$ atletas).

\section{Instrumentos}

Foi aplicado um questionário inicial, desenvolvido pelos pesquisadores, contendo informações pessoais como nome e idade, preferência de membro inferior (mediante autorrelato), data da menarca (se houvesse), bem como dados a respeito do tempo de treinamento do esporte e possíveis lesões prévias. Os dados antropométricos (massa corporal e estatura) foram mensurados por meio de balança e estadiômetro, respectivamente.

Para avaliação da capacidade funcional de membros inferiores (MMII), os seguintes testes foram aplicados pré e pós-intervenção:

- Single Leg Hop Test: Este teste foi utilizado para avaliar o desempenho funcional do membro inferior das atletas, bem como para identificar possíveis alterações durante a prática esportiva (SILVA; RIBEIRO; VENÂNCIO, 2010). As atletas foram orientadas a saltar o mais longe possível com os pés posicionados atrás de uma marca no chão, em apoio unipodal com a mesma perna (NEETER et al., 2006). Foi aplicada a versão modificada do teste, com livre movimentação dos braços objetivando maior semelhança com o gesto esportivo na sua execução (ZATTERSTROM et al., 2000). As jogadoras foram instruídas a realizar um pouso controlado e a distância foi medida em centímetros. Foram realizadas três repetições, com a primeira apenas para familiarização e foi considerada a média das outras duas tentativas (THOMEÉ et al., 2011). Houve um intervalo de descanso de 30 segundos entre as tentativas. Estudo prévio (ROSS; LANGFORD; WHELAN, 2002) descreveu um coeficiente de confiabilidade interclasse para o Single Hop Test de 0.92 - 0.96;

- Timed Hop Test: Para esse teste a variável utilizada foi o tempo. As atletas foram conduzidas a saltar o mais rápido possível até atingir uma distância de 6 metros, em apoio unipodal com a mesma perna, previamente determinada por uma linha reta no solo, marcada por fita (BOLGLA; KESKULA, 1997). Foi realizada uma tentativa com cada membro para familiarização e a média de outras duas tentativas foi considerada (THOMEÉ et al., 2011). Houve um intervalo de descanso de 30 segundos entre as tentativas. Ross, Langford e Whelan (2002) identificaram um coeficiente de confiabilidade interclasse para o Timed Hop Test de $0.66-0.92$.

- Side Hop Test: Esta ferramenta foi utilizada com objetivo de avaliar a estabilidade da articulação do tornozelo, bem como a potência de membros inferiores. Para a execução, as jogadoras foram posicionadas em apoio unipodal ao lado de duas linhas paralelas com $30 \mathrm{~cm}$ de distância, e foram instruídas a saltar lateralmente entre elas 10 vezes o mais rápido possível. Também foi permitida a livre movimentação dos braços. Foi realizado um teste de familiarização com cada membro e o resultado foi calculado por meio das médias de tempo para três tentativas em segundos (RABELLO et al., 2014). Houve um intervalo de descanso de 30 segundos entre as tentativas. Estudo prévio realizado por Kamonseki et al. (2018) identificou boa confiabilidade intra-avaliador e muito boa confiabilidade entre avaliadores no uso do Side hop para avaliar adolescentes.

Durante a execução de todos os testes as jogadoras receberam estímulo verbal por uma única avaliadora, previamente treinada.

\section{Intervenção}

O programa de prevenção de lesão consistiu de, aproximadamente, 20 minutos de exercícios antes do treino regular do esporte, 2 vezes por semana, durante 8 semanas, totalizando 16 sessões. O protocolo teve como base exercícios do Fifa11+ (SOLIGARD et al. 2008), exercícios funcionais (ACHENBACH et al., 2018), 
e exercícios de fortalecimento de quadril (SELKOWITZ; BENECK; POWERS, 2013), progredindo em relação ao nível de dificuldade a partir da $5 \underline{a}$ semana.

Inicialmente foram trabalhados exercícios de aquecimento (corrida em dupla com salto e contato ombro a ombro, corrida à volta do parceiro, evoluindo para sprints para a frente e para trás, corrida com joeIho para dentro), exercícios de força muscular (prancha ventral, ostra, ponte bipodal, Nordic Hamstring, evoluindo para prancha ventral alternando as pernas, prancha funcional com flexão de ombro tocando cone, ponte unipodal, exercício de fortalecimento do complexo glúteo em quatro apoios), pliometria (treino de aterrissagem/simulação de corrida, saltos laterais e frontais com agachamento, corrida lateral com agachamento bipodal, evoluindo para saltos alternados, salto sobre o cone arremessando a bola, saltos laterais com agachamento unipodal, corrida lateral com agachamento unipodal), e equilíbrio (arremesso de bola em apoio unipodal, apoio unipodal com os olhos fechados, evoluindo para apoio unipodal transpassando a bola pelo tronco e pernas, apoio unipodal desequilibrando o colega), podendo ser visualizados na Figura 1.

Figura 1 - Protocolo de exercícios realizados

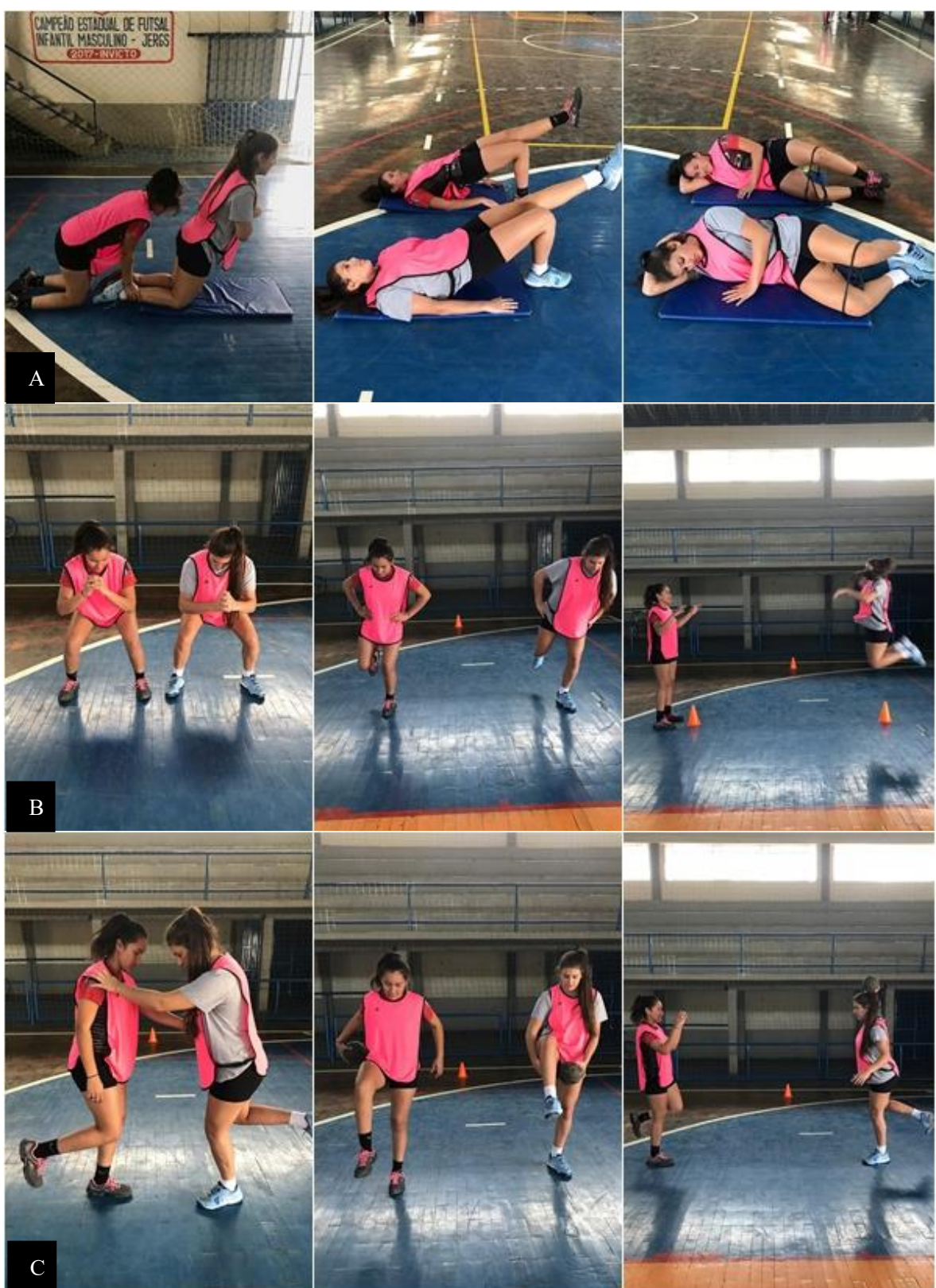

A: Exercícios de força (Nordic Hamstring, ponte unipodal e ostra); B: Exercícios de agilidade e pliometria (corrida lateral com agachamento bipodal, saltos laterais com agachamento unipodal, saltos sobre o cone arremessando a bola); C: Exercícios de equilíbrio (apoio unipodal desequilibrando o colega, apoio unipodal transpassando a bola pelo tronco e pernas, arremesso de bola em apoio unipodal).

Fonte: Os autores, 2019. 
Como principais diferenças entre os treinamentos do GC e do Gl, destaca-se que o Gl participou do programa preventivo, constando de três pilares principais: exercícios de estabilização lombopélvica, de agilidade/potência/pliometria e de equilíbrio, juntamente com o treinamento convencional de handebol, no qual foram abordados os fundamentos específicos do esporte, como passes e arremessos, a fim de melhorar o desempenho esportivo. Já o GC participou apenas dos exercícios relacionados aos fundamentos específicos da modalidade de handebol.

\section{Análise estatística}

Para a análise estatística utilizou-se o programa SPSS, versão 20.0, com análise descritiva, por meio de medidas de média e desvio padrão. Após a testagem da normalidade dos dados mediante o teste Shapiro-Wilk, indicou-se uma distribuição paramétrica. Dessa forma, as diferenças entre as testagens (pré e pós-testes), em ambos os grupos, foram avaliadas pelo teste $t$ de student pareado. Para comparação entre os dois grupos (GI e GC), tanto nos momentos pré-intervenção quanto no pós-intervenção, foi utilizado o test $t$ de student para amostras independentes. Também foi utilizado o test $t$ de student para amostras independentes para comparar as variáveis antropométricas entre os grupos ( $\mathrm{GC}$ e $\mathrm{Gl}$ ). Para todas as análises foi considerado um nível de significância de 0,05. Optamos por apresentar os resultados conforme a dominância de membro inferior, em conformidade com o estudo de Oliano et al. (2017).

\section{RESULTADOS}

A amostra foi constituída por 17 meninas pós-púberes, sendo 9 do GI com idade média de $13,67 \pm 1,50$ anos (massa corporal de $55,42 \pm 12,85 \mathrm{~kg}$ e estatura de $1,60 \pm 0,08 \mathrm{~m}$ ) e 8 do GC com média de idade de $13,38 \pm 1,18$ anos (massa corporal de 49,20 $\pm 6,17$ $\mathrm{kg}$ e estatura de $1,57 \pm 0,08 \mathrm{~m}$ ). Os grupos não diferiram quanto aos dados de caracterização amostral $(p>0,05)$, evidenciando homogeneidade entre eles.

As variáveis relacionadas com a capacidade funcional no membro inferior dominante, do Gl e do GC, pré e pós-intervenção, estão presentes na Tabela 1. Quanto à análise intragrupos, percebemos que houve uma melhora significativa em todas as variáveis no membro dominante do $\mathrm{Gl}$ ao final da intervenção $(p<0,05)$. Já no $G C$ houve melhora apenas no Single hop $(\mathrm{p}=0,001)$ e no Time hop $(\mathrm{p}=0,003)$, pós-intervenção. Na comparação intergrupos não houve diferença entre os grupos pré-intervenção, contudo o Gl apresentou melhores valores em relação ao $\mathrm{GC}$, nos testes Time hop $(\mathrm{p}=0,02)$ e Side hop $(\mathrm{p}=0,02)$, pós-intervenção.

A Tabela 2 mostra as variáveis relacionadas ao membro inferior não dominante, no qual foi possível destacar, na avaliação intragrupos, que o $\mathrm{Gl}$ apresentou melhora em todas as variáveis analisadas $(p<0,05)$, pós-intervenção, diferentemente do GC, que apresentou melhora apenas no Single hop $(p=0,001)$. As comparações intergrupos seguiram o mesmo padrão do membro dominante, ou seja, antes da intervenção

Tabela 1 - Comparação intra e intergrupos da capacidade funcional do membro inferior dominante pré e pós-intervenção

\begin{tabular}{|c|c|c|c|c|c|c|c|c|}
\hline Variáveis & & GI & & & GC & & & \\
\hline & PRE & PÓS & $p^{\prime}$ & PRÉ & PÓS & $p^{\prime}$ & $p^{\prime \prime}$ & $p^{\prime \prime \prime}$ \\
\hline Single hop $(\mathrm{cm})$ & $111,000 \pm 26,688$ & $160,000 \pm 18,179$ & $<0,001^{*}$ & $114,833 \pm 13,770$ & $149,666 \pm 18,157$ & $0,001^{*}$ & 0,707 & 0,245 \\
\hline Time hop (s) & $2,991 \pm 0,276$ & $2,164 \pm 0,258$ & $<0,001^{*}$ & $3,070 \pm 0,462$ & $2,525 \pm 0,333$ & $0,003^{*}$ & 0,666 & $0,021 *$ \\
\hline Side hop (s) & $11,516 \pm 4,019$ & $8,121 \pm 1,064$ & $0,019 *$ & $11,797 \pm 4,395$ & $11,086 \pm 3,458$ & 0,630 & 0,889 & $0,026^{*}$ \\
\hline
\end{tabular}

Gl= Grupo Intervenção, GC= Grupo Controle; $p^{\prime}=$ comparação intragrupos (pré x pós-teste), $p^{\prime \prime}=$ comparação intergrupos pré-intervenção, $p^{\prime \prime \prime}=$ comparação intergrupos pós-intervenção, dados expressos em média e desvio padrão * indica diferença significativa $(p<0,05)$.

Fonte: Os autores, 2019.

Tabela 2 - Comparação intra e intergrupos da capacidade funcional do membro inferior não-dominante pré e pós-intervenção

\begin{tabular}{|c|c|c|c|c|c|c|c|c|}
\hline Variáveis & & GI & & & GC & & & \\
\hline & PRE & PÓS & $p^{\prime}$ & PRÉ & PÓS & $p^{\prime}$ & $p^{\prime \prime}$ & $p^{\prime \prime \prime}$ \\
\hline Single hop $(\mathrm{cm})$ & $107,611 \pm 26,060$ & $159,611 \pm 13,355$ & $<0,001^{*}$ & $115,722 \pm 16,985$ & $149,166 \pm 18,069$ & $0,001^{*}$ & 0,446 & 0,182 \\
\hline Time hop (s) & $2,803 \pm 0,312$ & $2,101 \pm 0,261$ & $0,001^{*}$ & $2,977 \pm 0,518$ & $2,697 \pm 0,411$ & 0,182 & 0,400 & $0,002 *$ \\
\hline Side hop (s) & $11,571 \pm 4,091$ & $8,152 \pm 1,319$ & $0,012^{*}$ & $11,943 \pm 3,734$ & $12,045 \pm 4,948$ & 0,855 & 0,843 & $0,037^{*}$ \\
\hline
\end{tabular}

GI= Grupo Intervenção, GC= Grupo Controle; $p^{\prime}=$ comparação intragrupos (pré x pós-teste), p" = comparação intergrupos pré-intervenção, p’"' = comparação intergrupos pós-intervenção, dados expressos em média e desvio padrão, * indica diferença significativa $(p<0,05)$. 
os grupos não diferiram quanto às variáveis analisadas, porém pós-intervenção o Gl apresentou melhores escores nos testes Time hop $(p=0,002)$ e Side hop $(p=0,03)$.

\section{DISCUSSÃO}

Considerando que os testes funcionais aplicados no presente estudo são comumente usados como forma de triagem para identificar atletas com maior risco de lesão (GOOSSENS et al., 2015), posto que avaliam deficiências na função muscular (ERICSSON; DAHLBERG; ROOS, 2009), o presente trabalho trouxe resultados positivos da prática de um programa de prevenção de lesão sobre variáveis de desempenho funcional de membros inferiores em jovens atletas de handebol feminino. Sugere-se que a melhora obtida possa prevenir lesões futuras nessas atletas, uma vez que trabalhos ressaltam a eficácia da maioria dos exercícios utilizados neste estudo para a prevenção de lesão de membros inferiores em atletas (MAZUREK et al., 2018; SILVERS-GRANELLI et al., 2017; ELERIAN; EL-SAYYAD; DORGHAM, 2019; THOMPSON et al., 2017; WHITEHEAD et al., 2018; EUN-KYUNG, 2016).

Apesar de sua importância, os efeitos de programas preventivos voltados aos atletas jovens de handebol, principalmente do sexo feminino, ainda são pouco relatados na literatura. Indo ao encontro do nosso trabalho, Hammami et al. (2019) identificaram uma melhora significativa de componentes importantes do desempenho funcional de 21 atletas jovens de handebol feminino, como tempo de sprint, salto e equilíbrio, após a prática de um programa de treinamento pliométrico de nove semanas.

Outro estudo (CHAABENE et al., 2019a) também verificou a eficácia de um programa de treinamento pliométrico de oito semanas sobre o desempenho físico de jovens jogadoras de handebol. Vinte e uma atletas foram divididas em grupo intervenção (GI, $n=12$, idade= 15,9 anos), o qual realizou os exercícios pliométricos, e grupo controle $(\mathrm{GC}, \mathrm{n}=9$, idade= 15,9 anos), que manteve o treino regular do esporte. Nesse estudo os autores concluíram que o programa de treinamento pliométrico de curto prazo, durante a temporada de competição, melhorou a aptidão física das atletas (velocidade de mudança de direção, saltos e capacidade de corrida repetida).

Ademais, Achenbach et al. (2018) analisaram 23 times de handebol de ambos os sexos, sendo alocados em grupo intervenção $(\mathrm{Gl}, \mathrm{n}=168$, idade $=14,9)$ e grupo controle (GC, $n=111$, idade= 15,1). O Gl participou de um programa de prevenção de lesão, du- rante 10-12 semanas pré-temporada (com duração de 15 minutos e frequência de 2-3 vezes/semana), e uma vez na semana (com duração de 15 minutos) durante o período de competição. O programa era composto por exercícios semelhantes ao utilizado no presente estudo (força, pliometria e equilíbrio). Ao final, foi observado que a prática destes exercícios evitou lesões graves de joelho nesses atletas, ressaltando a importância da inclusão de protocolos preventivos no treinamento esportivo, bem como a conscientização dos técnicos das equipes.

Em uma revisão sistemática e metanálise de 11.773 atletas de futebol feminino (CROSSLEY et al. 2020) foi observado que o treinamento composto por variáveis como força, mobilidade e pliometria é capaz de reduzir as chances de lesões gerais de membros inferiores (27\%) e do ligamento cruzado anterior (45\%) em atletas do sexo feminino, contudo os autores destacam a importância de mais evidências com esta população.

O Nordic Hamstring, que é um exercício de fortalecimento excêntrico de isquiossurais, faz parte do programa preventivo Fifa 11+ para prevenção de lesão no esporte e foi utilizado no presente estudo, entre os exercícios de força muscular. Nesse contexto, Chaabene et al. (2019b) analisaram os efeitos de um treinamento de oito semanas deste exercício sobre componentes do desempenho funcional de 10 jovens jogadoras de handebol (15,9 anos de idade). 0 grupo intervenção (GI) realizou o exercício 2-3 sessões por semana em substituição a alguns exercícios específicos do handebol, enquanto o grupo controle (GC) seguiu o treinamento regular da equipe. Ao final da intervenção os autores observaram que o treinamento do GI foi mais eficaz do que o do GC na melhoria de aspectos relevantes para a performance em quadra, como o tempo de sprint, o salto, a mudança de direção e a capacidade de corrida repetida de jovens atletas de handebol.

A incapacidade dos atletas de manter o seu centro de massa sobre a base de suporte, tanto em pé quanto em movimento, tem sido discutida na literatura como um importante fator de risco intrínseco para o desenvolvimento de lesões nos membros inferiores. Os benefícios de programas preventivos são, na maioria das vezes, relacionados à modificação de fatores que incluem o equilíbrio estático e dinâmico. 0 estabelecimento regular do treinamento neuromuscular envolvendo o equilíbrio na rotina de treino dos atletas é visto como uma alternativa econômica frequentemente relatada, estando cada vez mais popular e demonstrando efeitos promissores (STEIB et al., 2016). 
Nesse contexto, Oliano et al. (2017) evidenciaram que a prática de 12 semanas do programa Fifa11+ associada ao treinamento convencional de handebol trouxe contribuições importantes sobre o equilíbrio postural de atletas jovens do sexo feminino com idades de 11 a 14 anos. Ainda, Steib et al. (2016) analisaram que um treinamento neuromuscular de no mínimo 6 semanas, com duração de 15 minutos 3 vezes por semana, interfere positivamente nas variáveis de equilíbrio estático e dinâmico que estão associadas às maiores chances de desenvolvimento de lesões de membros inferiores em atletas adultas de handebol feminino.

Apesar de no presente estudo não ter sido avaliado o equilíbrio das atletas, optamos por inserir, no protocolo, exercícios que também abordem essa variável, uma vez que estão associados à diminuição do risco de desenvolvimento de lesões, conforme exposto por Steib et al. (2016).

Percebemos que as variáveis do Side hop meIhoraram, pós-intervenção, apenas no GI (membro dominante $p=0,019$; membro não dominante $p=$ $0,012)$. Este teste pode ser utilizado tanto na avaliação da função de membros inferiores de indivíduos saudáveis quanto daqueles com lesões, e visa à avaliação da progressão do treinamento de atletas, pois mede as mudanças no desempenho funcional durante um programa preventivo ou de reabilitação (KAMONSEKI et al., 2018). Assim, as melhorias na força muscular, estabilidade articular e controle neuromuscular avaliadas neste teste podem estar relacionadas com os exercícios utilizados no protocolo aplicado no presente estudo.

Um programa de treinamento preventivo de 8 semanas associado à prática regular do handebol apresentado neste estudo demonstrou ganhos significativos nos aspectos desempenho funcional das atletas. Desse modo, sugere-se que tais ganhos possam interferir não só sobre os fatores de prevenção de lesão como também sobre os de performance das atletas em quadra, por aperfeiçoar a potência e agilidade dos membros inferiores.

Com base nesse aspecto, cabe destacar a importância de alertar os treinadores e preparadores físicos destas atletas a respeito da importância da inserção dos programas preventivos associados ao treino regular do handebol, conforme realizado por LaBella et al. (2011). Esses autores orientaram 45 treinadores a respeito da instauração de um programa de aquecimento neuromuscular de 20 minutos para atletas americanas de futebol e basquete feminino $(n=737)$ de escolas públicas de Ensino Médio. O programa uti- lizou exercícios semelhantes aos do presente estudo (fortalecimento progressivo, agilidade/ pliometria e equilíbrio), e os autores observaram que este método reduziu as lesões sem contato, de membros inferiores, das atletas.

O estudo realizado apresentou como limitações a perda amostral e a não realização de um cálculo amostral, para permitir resultados mais conclusivos.

\section{CONCLUSÕES}

Um período de 8 semanas de treinamento de um programa preventivo, incluindo exercícios de aquecimento, força, agilidade, pliometria e equilíbrio, melhorou significativamente a capacidade funcional de membros inferiores em jovens atletas de handebol feminino. Esses achados indicam que este tipo de intervenção é capaz de modificar positivamente os fatores de risco para o desenvolvimento de lesões de membros inferiores destas atletas.

Sugere-se a realização de mais estudos com essas atletas, para permitir identificar os efeitos neuromusculares deste tipo de treinamento, a curto e a longo prazos, bem como a maior conscientização tanto das atletas quanto dos treinadores a respeito da importância da prática preventiva associada ao treino regular do esporte.

\section{REFERÊNCIAS}

ACHENBACH, L.; KRUTSCH, V.; WEBER, J.; NERLICH, M.; LUIG, P.; LOOSE, O.; ANGELE, P.; KRUTSCH, W. Neuromuscular exercises prevent severe knee injury in adolescent team handball players. European Society of Sports Traumatology, Knee Surgery, Arthroscopy, v. 26, n. 7, p. 1.901-1.908, 2018.

BOLGLA, L. A.; KESKULA, D. R. Reliability of lower extremity functional performance tests. $J$ Orthop Sports Phys Ther., v. 26, n. 3, p. 138-142, 1997.

CHAABENE, H.; NEGRA, Y.; MORAN, J.; PRIESKE, O.; SAMMOUD, S.; CAMPILLO-RAMIREZ, R.; GRANACHER, U. Effects of an Eccentric Hamstrings Training on Components of Physical Performance in Young Female Handball Players. Int $\mathrm{J}$ Sports Physiol Perform. 2019a. [Epub ahead of print].

CHAABENE, H.; NEGRA, Y.; MORAN, J.; PRIESKE, O.; SAMMOUD, S.; CAMPILLO-RAMIREZ, R.; GRANACHER, U. Plyometric Training Improves Not Only Measures of Linear Speed, Power, and Change-of-Direction Speed But Also Repeated Sprint Ability in Female Young Handball Players. J Strength Cond Res. 2019b. [Epub ahead of print].

CROSSLEY, K. M.; PATTERSON, B. E.; CULVENOR, A. G.; BRUDER, A. M.; MOSLER, A. B.; MENTIPLAY, B. F. Making football safer for women: a systematic review and meta-analysis of 
injury prevention programmes in 11773 female football (soccer) players. Br J Sports Med., 2020. [Epub ahead of print].

DIFIORI, J. P.; BENJAMIN, H. J.; BRENNER, J. S.; GREGORY, A.; JAYANTHI, N.; LANDRY, G. L.; LUKE, A. Overuse injuries and burnout in youth sports: a position statement from the American Medical Society for Sports Medicine. Clin J Sport Med., v. 24, n. 3, p. 3-20, 2014.

ELERIAN, A. E.; EL-SAYYAD, M. M.; DORGHAM, H. A. A. Effect of Pre-training and Post-training Nordic Exercise on Hamstring Injury Prevention, Recurrence, and Severity in Soccer Players. Ann Rehabil Med., v. 43, n. 4, p. 465-73, 2019.

ERICSSON, Y. B.; DAHLBERG, L. E.; ROOS, E. M. Effects of functional exercise training on performance and muscle strength after meniscectomy: a randomized trial. Scand J Med Sci Sports, v. 19, n. 2, p. 156-165, 2009.

EUN-KYUNG, K. I. M. The effect of gluteus medius strengthening on the knee joint function score and pain in meniscal surgery patients. J. Phys. Ther. Sci., v. 28, n. 10, p. $2.751-$ 2.753, 2016.

GIROTO, N.; HESPANHO JUNIOR, L. C.; GOMES, M. R.; LOPES, A. D. Incidence and risk factors of injuries in Brazilian elite handball players: A prospective cohort study. Scand J Med Sci Sports, v. 27, n. 2, p. 195-202, 2017.

GOOSSENS, L.; WITVROUW, E.; VANDEN BOSSCHE, L.; CLER$C Q, D$. D. Lower eccentric hamstring strength and single leg hop for distance predict hamstring injury in PETE students. Eur J Sport Sci., v. 15, n. 5, p. 436-442, 2015.

HAMMAMI, M.; CAMPILLO-RAMIREZ, R.; GAAMOURI, N.; ALOUI, G.; SHEPHARD, R. J.; CHELLY, M. S. Effects of a Combined Upper- and Lower-Limb Plyometric Training Program on High-Intensity Actions in Female U14 Handball Players. Pediatr Exerc Sci., v. 31, n. 4, p. 1-8, 2019. [Epub ahead of print].

KAMONSEKI, D. H.; CEDIN, L.; TAVARES-PRETO, J.; CALIXTRE, L. B. Reliability, validity, and minimal detectable change of Side Hop Test in male children and adolescents. Phys Ther Sport, v. 34, p. 141-147, 2018.

LABELLA, C. R.; HUXFORD, M. R.; GRISSOM, J.; KIM, K. Y.; PENG, J.; CHRISTOFFEL, K. K. Effect of neuromuscular war$\mathrm{m}$-up on injuries in female soccer and basketball athletes in urban public high schools: cluster randomized controlled trial. Arch Pediatr Adolesc Med., v. 165, n. 11, p. 1.0331.040, 2011.

MAZUREK, K. L.; ZMIJEWSKI, P.; MAKARUK, H.; MRÓZ, A.; LIPNSKA, P. Effects of Short-Term Plyometric Training on Physical Performance in Male Handball Players. Journal of Human Kinetics, v. 63, p. 137-148, 2018.

NEETER, C.; GUSTAVSSON, A.; THOMÉE, P.; AUGUSTSSON, J.; THOMÉE, R.; KARLSSON, J. Development of a strength test battery for evaluating leg muscle power after anterior cruciate ligament injury and reconstruction. Knee Srug Sports Traumatol Arthrosc, v. 14, n. 6, p. 571-580, 2006.
OLIANO, V. J.; TEIXEIRA, L. P.; LARA, S.; BALK, R. S.; FAGUNDES, S. G. Effect of FIFA $11+$ in addition to conventional handball training on balance and isokinetic strength. Rev. Bras. Cineantropom Desempenho Hum., v. 19, n. 4, p. 406415, 2017.

OLIVEIRA, R. Lesões nos jovens atletas: conhecimento dos factores de risco para melhor prevenir. Revista Portuguesa de Fisioterapia no Desporto, v. 3, n. 1, p. 33-38, 2009.

RABELLO, L. M.; MACEDO, C. S. G.; OLIVEIRA, M. R.; FREGUETO, J. H.; CAMARGO, M. Z.; LOPES, L. D.; SHIGAKI, L.; GIL, A. W.; KAMUZA, C. Relação entre testes funcionais e plataforma de força nas medidas de equilíbrio em atletas. Rev. Bras. Med. Esporte, v. 20, n. 3, p. 219-222, 2014.

ROSS, M. D.; LANGFORD, B.; WHELAN, P. J. Test-retest reliability of 4 single-leg horizontal hop tests. J Strength Cond Res., v. 16, n. 4, p. 617-622, 2002.

SELKOWITZ, D. M.; BENECK, G. J.; POWERS, C. M. Which Exercises Target the Gluteal Muscles While Minimizing Activation of the Tensor Fascia Lata? Electromyographic Assessment Using Fine-Wire Electrodes. J Orthop Sports Phys Ther, v. 43, n. 2, p. 54-64, 2013.

SILVA, T.; RIBEIRO, F.; VENÂNCIO, J. Comparação da performance funcional do membro inferior entre jovens futebolistas e jovens não treinados. Fisioter. Mov., v. 23, n. 1, p. 105-112, 2010.

SILVERS-GRANELLI, H. J.; BIZZINI, M.; ARUNDALE, A.; MANDELBAUM, B. R.; SNYDER-MACKLER, L. Does the FIFA 11+ Injury Prevention Program Reduce the Incidence of ACL Injury in Male Soccer Players? Clin Orthop Relat Res, v. 475, n. 10, p. 2.447-2.455, 2017.

SOLIGARD, T.; MYKLEBUST, G.; STEFFEN, K.; HOLME, I.; SILVERS, H.; BIZZINI M.; JUNGLE, A.; DVORAK, J.; BAHR, R.; ANDERSEN, T. E. Comprehensive warm-up programme to prevent injuries in young female footballers: cluster randomized controlled trial. BMJ, p. 1-9, 2008.

SPIESZNY, M.; ZUBIK, M. Modification of Strength Training Programs in Handball Players and its Influence on Power During the Competitive Period. Journal of Human Kinetics, v. 63, p. 149-160, 2018.

STEFFEN, K.; NILSTAD, A.; KRISTIANSLUND, E. K.; MYKLEBUST, G.; BAHR, R.; KROSSHAUG, T. Association between lower extremity muscle strength and noncontact $\mathrm{ACL}$. Injuries Med Sci Sports Exerc., v. 48, n. 1, p. 2.082-2.089, 2016.

STEIB, S.; ZAHN, P.; EULENBURG, C. Z.; PFEIFER, K.; ZECH, A. Time-dependent postural control adaptations following a neuromuscular warm-up in female handball players: a randomized controlled trial. BMC Sports Sci Med Rehabil., v. 8, n. 33, 2016.

THOMEÉ, R.; KAPLAN, Y.; KVIST, J.; MYKLEBUST, G.; RISBERG, M. A.; THEISEN, D.; TSEPIS, E.; WERNER, S.; WONDRASCH, B.; WITVROUW, E. Muscle strength and hop performance criteria prior to return to sports after $\mathrm{ACL}$ reconstruction. Knee Surg Sports Traumatol Arthrosc., v. 19, n. 11, p. 1.7981.805, 2011. 
THOMPSON, J. A.; TRAN, A. A.; GATEWOOD, C. T.; SHULTZ, R.; SILDER, A.; DELP, S. L.; DRAGOO, J. L. Biomechanical Effects of an Injury Prevention Program in Preadolescent Female Soccer Athletes. Am J Sports Med., v. 45, n. 2, p. 294-301, 2017.

WAGNER, H.; FINKENZELLER, T.; WÜRTH, S.; VONDUVILLARD, S. P. Individual and Team Performance in Team-Handball: A Review. J Sports Sci Med. v. 13, n. 4, p. 808-816, 2014.

WHITEHEAD, M. T.; SCHEETT, T. P.; MCGUIGAN, M. R.; MARTIN, A. V. A Comparison of the Effects of Short-Term Plyometric and Resistance Training on Lower Body Muscular Performance. J Strength Cond Res., v. 32, n. 10, p. 2.7432.749, 2018.

ZATTERSTROM, R.; FRIDEN, T.; LINDSTRAND, A.; MORITZ, $U$. Rehabilitation following acute anterior cruciate ligament injuries - a 12-month follow-up of a randomized clinical trial. Scand J Med Sci Sports., v. 10, n. 3, p. 156163, 2000. 